\title{
Meticulous History and Examination: Still Remains the Backbone of Diagnosis
}

\author{
Urvashi Sharma ${ }^{1}$, Umang Juneja², Swati Garg ${ }^{3}$
}

\begin{abstract}
A high degree of suspicion and an ectopic conscious clinician is required for diagnosis of ectopic pregnancy. Only when you suspect it, a detailed history of the patient and a meticulous examination will reveal the presence of an ectopic pregnancy, which can then be confirmed by the appropriate bedside investigations. The cases of ectopic pregnancy where the diagnosis is delayed or, which are misdiagnosed, carry a high morbidity and mortality, as the morbidity and mortality associated with ectopic pregnancy is directly related to the length of time required for diagnosis. We report three such cases of ectopic pregnancy where the diagnosis was delayed due to variable and unpredictable presentations, in whom the initial history and examination was missed as the presence of ectopic pregnancy was not suspected by the presentation.

Keywords: Ectopic pregnancy, Misdiagnosed, Pelvic inflammatory disease, Tubal corrective surgery.

Journal of Mahatma Gandhi University of Medical Sciences \& Technology (2019): 10.5005/jp-journals-10057-0103
\end{abstract}

\section{INTRODUCTION}

Ectopic pregnancy (EP) is implantation of a fertilized ovum in an area other than the endometrial lining of the uterus. The commonest site is ampullary portion of the fallopian tube (70\%); rest uncommon sites are isthmic (12\%), fimbrial (11\%), interstitial (2-3\%), ovarian (3\%), and rarely cervical and abdominal., ${ }^{1,2}$ Earliest rupture occurs in isthmic portion of the tube (6 weeks), then in ampullary (8-10 weeks) and interstitial (3-4 months). ${ }^{3}$

It is potentially lethal as it accounts for $3.5-7.1 \%$ of all pregnancyrelated deaths in India. ${ }^{4}$ Without prompt diagnosis and treatment, it can lead to significant maternal morbidity and mortality. Despite significant advances, it remains the leading cause of maternal death in 1st trimester.

Its incidence is $1.5-2 \%$ of all pregnancies. Ectopic pregnancy rates increased almost fourfold (from 4.5 per 1,000 to 16.8 per 1,000 pregnancies since 1970). Incidence is rising because of improved diagnosis and rise in risk factors, which are those causing delayed transport of the fertilized ovum through the fallopian tubes. The main risk factor being previous $\mathrm{EP}$, pelvic inflammatory disease (PID), tubal corrective surgery or tubal sterilization, presence of intrauterine device, and use of assisted reproductive technology.

The clinical presentation is variable, and a high degree of suspicion is required during history and clinical examination. The classical triad is present in $50 \%$ cases with ruptured EP, pain (most constant feature-95\%), is variable in severity and amenorrhea (60-80\%), delayed period or slight spotting at expected menses and vaginal bleeding (70\%), which can be scanty dark brown. Others symptoms may be nausea, vomiting, fainting, and syncopal attacks (10\%) due to reflex vasomotor disturbances; may have previous attack of acute pain from which she has recovered; and bladder and bowel complaints.

Clinical presentation is variable and again a high degree of suspicion and ectopic conscious clinician is required for diagnosis. Abdomen may be tense and tender, per speculum reveals minimal bleeding and per vaginal examination (which should be done gently) reveals cervical excitation, uterus may be soft, normal size, may be bulky and deviated, fornix will be tender or mass may be felt in the fornix, and pouch of Douglas may be full.
${ }^{1-3}$ Department of Obstetrics and Gynecology, Mahatma Gandhi Medical College and Hospital, Jaipur, Rajasthan, India

Corresponding Author: Swati Garg, Department of Obstetrics and Gynecology, Mahatma Gandhi Medical College and Hospital, Jaipur, Rajasthan, India, Phone: +919414048000, e-mail: drswatigarg@ mgumst.org

How to cite this article: Sharma U, Juneja U, Garg S. Meticulous History and Examination: Still Remains the Backbone of Diagnosis. J Mahatma Gandhi Univ Med Sci Tech 2019;4(2):49-52.

Source of support: Nil

Conflict of interest: None

On investigation in suspected cases, urine pregnancy test (UPT) is positive in $95 \%$ cases, serum $\beta$-hCG shows a minimum rise by $66 \%$ every 48 hours (indicates viable intrauterine pregnancy), serum progesterone-usually less than 5-10 ng/mL. Ultrasonographytransvaginal, transabdominal and color Doppler are helpful depending upon the site of EP. Culdocentesis is positive in $70-90 \%$ cases of ruptured EP. Laparoscopy is the gold standard as it is both diagnostic and therapeutic.

Transvaginal sonography (TVS) detects intrauterine gestations as early as 1 week after the missed period. The discriminatory level of $\beta$-hCG is $1500 \mathrm{IU} / \mathrm{L}$; if the levels are more than $1500 \mathrm{IU} / \mathrm{L}$ with an empty uterus on TVS, it is nearly $100 \%$ diagnostic of EP.

We report three such cases of EP where the diagnosis was delayed due to variable and unpredictable presentations, in whom the initial history and examination was missed as the presence of EP was not suspected.

\section{Case Description}

\section{Case 1}

A 35-year-old para 3 live 3, married Hindu female of low socioeconomical group, reported to casualty at $9 \mathrm{pm}$ in evening, with chief complaints of severe pain lower abdomen, nausea, and vomiting since 1 day. 
Pain was sudden, generalized, dull aching, all over abdomen, no radiating and no referred pain. It was associated with one to two episodes of vomiting. There was no history of fever, abdominal distention, diarrhea, constipation, discharge or bleeding per vaginum/rectum, urinary complains, trauma, or any other abdominal complaints. No h/o weakness and dizziness. Past medical history was insignificant; she had sterilization done 4 years ago and her last menstrual period (LMP) was 1 month ago with previous regular periods. Her family and personal history was noncontributory.

On general physical examination, she was conscious, cooperative, well oriented to time, place, and person, pulse was 128 /minute, regular, no radioradial delay or radiofemoral delay, $\mathrm{BP}$ was $96 / 50 \mathrm{~mm} \mathrm{Hg}$ (right arm in sitting position), $\mathrm{SpO}_{2} 96 \%$, RR 20/minute, Temp $98.4^{\circ} \mathrm{F}$ (axillary), BMI $20.8 \mathrm{~kg} / \mathrm{m}^{2}$, pallor was significant, no icterus and cyanosis, orodental hygiene was average, no neck swelling or dilated veins, breast was normal, there was no lymphadenopathy, clubbing, or edema. Systemic examination was normal; abdominal palpation revealed tenderness in the lower quadrant.

The patient was admitted in casualty under medicine department with provisional diagnosis of severe anemia and acute abdomen. Initial resuscitation done and patient was started on venotonics, IV fluids, and antibiotics. All investigations along with crossmatch sent. USG whole abdomen advised.

Initial investigations revealed: $\mathrm{Hb}: 6.4 \mathrm{~g} / \mathrm{dL}, \mathrm{TSH}: 1.84 \mathrm{mlU} / \mathrm{mL}$, RBS: 200 mg\%, HbA1c: 5.5\%, viral markers: negative, urine routine/ LFT/RFT/serum electrolytes: within normal limits, coagulation profile: PT—18.700 seconds, INR - 1.508, ESR—2, USG—mild ascites seen, no other abnormality detected. Uterus and adnexa couldn't be commented as bladder was not full.

Surgery reference was done, in view of severe pain abdomen and ascites. The patient was found to have severe anemia and generalized tenderness in the lower quadrant. Serum amylase, lipase, and repeat CBC ordered-found normal except $\mathrm{Hb} 4.2 \mathrm{~g} /$ $\mathrm{dL}$, with neutrophilia $82 \%$. Review USG was ordered and till then conservative management was started.

The case was referred to the gynecology department next morning, as her lower pain abdomen was not relieved. Gynec examination revealed soft and posterior cervix, with cervical motion tenderness, uterus was anteverted and anteflexed, soft, mobile, 6 weeks in size, and there was fullness and tenderness present in bilateral adnexa. On the basis of examination, the patient was advised UPT, which was positive. With the provisional diagnosis of EP, patient was shifted to the gynecology department for further investigation and management. Review abdominal, followed by vaginal ultrasound advised; uterus was normal with a heterogeneous lesion of approx. $40 \times 56 \mathrm{~mm}$ seen in left adnexa with no definite internal vascularities, s/o left adnexal heterogeneous mass lesion. Significant free fluid in the abdominal cavity? Ascites? Hemoperitoneum?

With the provisional diagnosis of 35-year-old G4 P3 L3 with? Left ruptured EP with failed sterilization, patient was planned for emergency laparotomy. After counseling and consent, laparotomy was done with all resuscitative measures. A left ruptured tubal EP along with $1.5 \mathrm{~L}$ of blood and clots seen in the abdominal cavity. Left-side Falope's ring seen in left mesosalpinx and right ring was normally placed. Left salpingectomy and right repeat tubal sterilization were done. The postoperative period was uneventful and the patient was discharged on day 7 of surgery.

\section{Case 2}

A 30-year-old married Hindu female of middle socioeconomical group, housewife, reported to casualty with complaints of severe pain in lower abdomen, shortness of breath, generalized body weakness, and dizziness and vomiting, all since 2 days.

Pain was sudden, generalized, dull aching, and present all over abdomen, without radiating or referred pain. Shortness of breath was sudden in onset, not associated with cough and positional change, no relieving or aggravating factors. The patient also complained of multiple episodes of vomiting, mainly containing food particles, nonprojectile, not blood-stained, and not related to food intake. No h/o fever, abdominal distention, diarrhea, constipation, bloating, bleeding per rectum, urinary complains, trauma, or any other abdominal/respiratory complaints were noted.

Past medical and surgical, family, and personal history was normal. She has three live issues and not using any contraceptive. Her LMP was 5 days back but on detailed history she said that she has taken I-pill in her previous cycle.

General physical examination revealed: pulse-119/minute, regular, no radioradial delay or radiofemoral delay, BP-100/70 $\mathrm{mm} \mathrm{Hg}, \mathrm{SpO}_{2}-88 \%$ on room air, $99 \%$ on oxygen, $\mathrm{RR}-24 /$ minute, temp-98.2 ${ }^{\circ} \mathrm{F}, \mathrm{BMI}-24.6 \mathrm{~kg} / \mathrm{m}^{2}$, pallor was significant.

The CVS and CNS examination was normal. On auscultation of lungs, bilateral air entry was decreased with course crepitation. No tracheal shift seen, equal expansion of chest seen. Abdominal examination was normal. On investigation, hemoglobin was $3.6 \mathrm{~g} /$ $\mathrm{dL}$; other initial investigations were normal.

Chest X-ray showed bilateral pleural effusion, no cardiomegaly. The USG revealed bilateral pleural effusion (right $>$ left) along with mild ascites. The USG pelvis was not advised as the patient was carrying the same done 2 weeks back, showing normal uterus and adnexa.

The patient was admitted in ICU under medical department with provisional diagnosis of 30-year-old female P3 L3 with acute respiratory distress syndrome (ARDS) with severe anemia with sepsis, and was started on ventilator support and IV antibiotics. About 4 units of blood transfused in course of ICU stay of 2 daysfollowing which $\mathrm{Hb}$ came to $8.4 \mathrm{~g} / \mathrm{dL}$. Spirometry and chest physiotherapy were done. Surgery reference done in view of ascites; they advised X-ray flat plate abdomen, which was normal. Patient's condition didn't improve even after 2 days of management and on further evaluation the patient was suspected to have stress cardiomyopathy. 2-D echo, troponin I, and cardiology reference were advised, which were normal.

After 2 days, gynecology reference was done in view of bleeding per vaginum and anemia. Upon further probing her history, she gave history of MTP pill (not I-Pill) intake 7 days ago following which she developed bleeding per vaginum since last 5 days. Menstrual history revealed that her LMP was 5 days back, which occurred after amenorrhea of $1 \frac{1}{2}-2$ months. She has two live issues and was not using any contraceptive method.

Genital examination revealed slight bleeding per vaginum, and bimanual examination showed soft and posterior cervix, uterus was anteverted and anteflexed soft, mobile, normal size, left adnexa-fullness and tenderness present, right adnexa-NAD following which UPT was advised, was positive and a provisional diagnosis of 30-year-old G4 P3 L3, severe anemia with? Intrauterine retained product of conception? EP? Threatened abortion was made. 
The USG pelvis was advised, which showed normal uterus with left-side tubo-ovarian mass $(48 \times 49 \mathrm{~mm})$. Ectopic pregnancy with no to minimal vascularity. Free fluid seen in pouch of Douglas with some fine echoes. $\beta$-hCG (in dilution) was done, which was $310.9 \mathrm{mIU} / \mathrm{mL}$.

With final diagnosis of 30-year-old G4 P3 L3 with ruptured left EP with severe anemia, immediate laparoscopic left salpingectomy was done with all resuscitative measures (patient refused for sterilization). The postoperative period was uneventful and the patient was discharged on day 5 of surgery.

\section{Case 3}

A 34-year-old P2 L2 married Hindu female, housewife, belonging to lower socioeconomic class, presented in emergency, after being referred from district Hospital, Karauli, with history of fall from bike, 5 hours back. The patient was attended by the surgery resident on duty. Patient presented with pain and abdominal distention since the time of accident. Pain was acute and generalized, not radiating or referred to any site. There was no h/o fever, nausea, vomiting, diarrhea, constipation, urinary complains, discharge or bleeding per rectum, or any other abdominal complains.

Past medical, surgical, family, and personal history was normal. She was on day 3 of menstrual period with regular cycles and had two live issues.

General physical examination revealed significant pallor with pulse of 116/minute, BP-90/66 $\mathrm{mm} \mathrm{Hg}$. Other examination was normal except slight distention and tenderness of lower abdomen.

Patient admitted in ICU under the surgery department with provisional diagnosis of 34-year-old female with blunt trauma abdomen due to road traffic accident. On investigation, hemoglobin was $3.4 \mathrm{~g} / \mathrm{dL}$; other initial investigations were normal. Ultrasonography showed free fluid in all quadrants and abdomen. Hemoperitoneum, no other abnormality detected.

Initial resuscitation was done; blood and blood products were arranged. After informed consent, patient was shifted to OT for exploratory laparotomy in view of blunt trauma abdomen. Prior to laparotomy, 4 quadrant tapping was done in OT, which was negative and hence surgery was cancelled and she was again shifted to ICU.

Following which an urgent gynecology reference was done. On gynec evaluation, the patient gave history of bleeding per vaginum since 3 days after an amenorrhea of $1 \frac{1}{2}$ months. On further asking, the patient gave history of UPT, which was faintly positive 6 days ago following which she took MTP pill without prescription.

On local genital examination, per vaginal bleeding was present, cervix was soft and anterior, uterus reteroverted and retroflexed, slightly bulky, left fornix fullness present, tender, right fornix free and nontender. The UPT was done bedside which was positive; therefore, culdocentesis was done in ICU, which revealed hemoperitoneum.

With final diagnosis of 34-year-old G3 P2 L2 with $1 \frac{1}{2}$ month of amenorrhea with left ruptured EP with $\mathrm{h} / \mathrm{o}$ road traffic accident with severe anemia. Laparotomy was done. A left-side ruptured ectopic was seen. Around $1 \mathrm{~L}$ of hemoperitoneum along with 1500 cc of clots was noted. Left salpingectomy was done followed by gut exploration by the surgeon in view of blunt trauma abdomen, which was found normal. The postoperative period was uneventful and the patient was discharged on day 4 of surgery.

\section{Case Summary}

\section{Case 1}

- Provisional diagnosis-acute abdomen with severe anemia and ascites

- Final diagnosis -intra-abdominal hemorrhage due to ruptured EP in a patient of failed sterilization

\section{Case 2}

- Provisional diagnosis-ARDS with severe anemia and sepsis with? stress cardiomyopathy

- Final diagnosis - Intra-abdominal hemorrhage due to ruptured EP following MTP pill intake

\section{Case 3}

- Provisional diagnosis-blunt trauma abdomen due to road traffic accident

- Final diagnosis-intra-abdominal hemorrhage due to ruptured EP following MTP pill intake

\section{Discussion}

There is a need of accurate and timely diagnosis in cases of EP as morbidity and mortality associated with EP is directly related to the length of time required for diagnosis. In Centre for Disease Control and Prevention $(C D C)$ survey, two-thirds of all patients who were later proven to have EP were previously seen by a physician, and either the diagnosis was deferred or the condition was incorrectly assessed. ${ }^{5}$ High degree of suspicion is the key for prompt diagnosis and timely management of EP.

The diagnosis often presents great difficulty and is usually missed because it is not suspected. It is well described by McFadyen in 1981: "Pregnancy in the fallopian tube is a black cat on a dark night. It may make its presence felt in subtle ways and leap at you or it may slip past unobserved. Although it is difficult to distinguish from cats of other colors in darkness, illumination clearly identifies it." ${ }^{6}$ Why does the clinician is deceived? He either does not suspect pregnancy at all or thinks that the patient is normally pregnant or is aborting a normal pregnancy and also because there is nothing characteristic about the presentation as pain is extremely variable in intensity, location, and character; amenorrhea may be absent in a fourth of women; adnexal mass in only up to $50 \%$ women; and cervical motion tenderness may not be present.

The only way to diagnose the EP is to be "ectopic minded," that is, to keep a high index of suspicion about its presence, and with that in mind, the history and examination will give a clue to the diagnosis, as was done in these cases.

Management of EP depends upon patient's clinical status, age, parity, size and site of ectopic, desire for future fertility, and history of prior surgery. The aim has changed from "saving the mother's life" to "saving the woman's fertility." The management options are expectant, medical, or conservative and radical surgery depending on the case.

Few rare sites of EP, which are now emerging with modern obstetrics, need special mention here: Cesarean scar ectopic (implantation within the myometrium of a prior cesarean delivery scar with incidence of 1:2000 normal pregnancies). 
A heterotopic pregnancy involves coexisting pregnancies at two different implantation sites. The most common combination is an intrauterine and an extrauterine gestation.

Ectopic pregnancy after hysterectomy is also not an uncommon event with its frequency increasing since it being reported for the first time in 1895 . Since then, around 72 cases of post-hysterectomy EP were reported. ${ }^{7}$ Ectopic placental implantations in abdominal pregnancies have been described in various case reports that include the omentum, spleen, liver, and retroperitoneum, among others. $^{8-11}$

Misdiagnosis of EP is very frequent especially if the patient present in any department other than gynecology leading to delay in diagnosis. A collaborative study published by PGI, Chandigarh, and Government Medical College, Chandigarh, stated that $27 \%$ (78 cases out of total of 282) were misdiagnosed before a correct diagnosis was made. ${ }^{12}$ A study conducted by Dickens et al. highlights the ethical and legal issues related to EP and emergency care. According to this, those who undertake or counsel deliberate delay of medically indicated treatment can be charged with criminal offences and civil (noncriminal) liability and medical professional misconduct. ${ }^{13}$ In 2003, the Supreme Court in New York awarded a compensation of $\$ 175,000$ when there was an intra-abdominal bleed because of rupture attributed to delay in diagnosing EP. ${ }^{14}$ Similarly, in a study from South Wales a compensation of $£ 15,000$ was given for failure to diagnose EP.

\section{SUMMARY}

Incidence of EP is rising. It can be diagnosed early with meticulous history and thorough clinical examination. The management strategy has shifted from ablative surgery to conservative fertility preserving therapy and laparoscopy/laparotomy should be done when in doubt. Choice today is laparoscopic conservative surgery for unruptured EP.

\section{Take-home Message}

Ectopic pregnancy should be ruled out in all women of reproductive age presenting with acute abdomen, irrespective of their menstrual cycle day, are sterilized, using contraceptives, marital status, and even if they are hysterectomized. Before prescribing an MTP pill, ensure that the pregnancy is intrauterine. The physician who is "ectopic minded" rarely fails to make the diagnosis.

\section{References}

1. Atrash HK, Friede A, Hogue CJ. Abdominal pregnancy in the United states: Frequency and maternal mortality. Obstet Gynecol 1987; 69(3 Pt 1):333.

2. Worley KC, Hnat MD, Cunningham FG. Advanced extrauterine pregnancy: diagnostic and therapeutic challenges. Am J Obstet Gynecol 2008;198(3):297e1. DOI: 10.1016/j.ajog.2007.09.044.

3. Ash A, Smith A, Maxwell D. Caesarean scar pregnancy. BJOG 2007;114(3):253. DOI: 10.1111/j.1471-0528.2006.01237.x.

4. Tahmina S, Daniel M, Solomon P. Clinical analysis of ectopic pregnancies in a tertiary care centre in southern India: a six year retrospective study. J Clin Diagn Res 2016;10(10):QC13-QC16. DOI: 10.7860/JCDR/2016/21925.8718.

5. Jones III HW. Te Linde's operative gynaecology. Ectopic Pregnancy. Wolters Kluwer; 2015. p. 775.

6. McFadyen IR. Clin Obstet Gynaecol 1981 Lozeau AM, Potter B. Diagnosis and management of ectopic pregnancy. Review article. Am Fam Physician 2005;72(9):1707-1714.

7. Flystra DL. Ectopic pregnancy after hysterectomy may not be so uncommon: a case report and review of literature. Case Reports in Women's Health 2015;7:8-11. DOI: 10.1016/j.crwh.2015.04.001.

8. Chin PS, Wee HY, Chern BS. Laparoscopic management of primary hepatic pregnancy. Aust N Z J Obstet Gynaecol 2010;50(1):95. DOI: 10.1111/j.1479-828X.2009.01113.x.

9. Chopra S, Keepanasseril A, Suri V, et al. Primary omental pregnancy: case report and review of literature. Arch Gynecol Obstet 2009;279(4):441. DOI: 10.1007/s00404-008-0730-4.

10. Gang G, Yudong Y, Zhang G. Successful laparoscopic management of early splenic pregnancy: case report and review of literature. J Minim Invasive Gynecol 2010;17(6):794. DOI: 10.1016/j.jmig.2010.07.004.

11. Martínez-Varea A, Hidalgo-Mora JJ, Payá V, et al. Retroperitoneal ectopic pregnancy after intrauterine insemination. Fertil Steril 2011;95(7):2433.e1. DOI: 10.1016/j.fertnstert.2011.02.026.

12. Saha PK, Gupta P, Goel P, et al. Ectopic pregnancy: a diagnostic dilemma. Int J Reprod Contracept Obstet Gynecol 2016;5(2):367-370

13. Dickens BM. Ectopic pregnancy and emergency care: ethical and legal issues. Int J Gynecol Obstet 2003;82(1):121-126. DOI: 10.1016/ s0020-7292(03)00175-9.

14. Laska LL. Rupture attributed to delay in diagnosing ectopic pregnancy. Med Malpract Verd, Settlem Exp 2003;15(5):96-104. 